\title{
Provably Repairing the ISO/IEC 9798 Standard for Entity Authentication
}

\author{
David Basin, Cas Cremers, and Simon Meier \\ Institute of Information Security \\ ETH Zurich, Switzerland
}

\begin{abstract}
We formally analyze the family of entity authentication protocols defined by the ISO/IEC 9798 standard and find numerous weaknesses, both old and new, including some that violate even the most basic authentication guarantees. We analyse the cause of these weaknesses, propose repaired versions of the protocols, and provide automated, machinechecked proofs of the correctness of the resulting protocols. From an engineering perspective, we propose two design principles for security protocols that suffice to prevent all the weaknesses. Moreover, we show how modern verification tools can be used for falsification and certified verification of security standards. The relevance of our findings and recommendations has been acknowledged by the responsible ISO working group and an updated version of the standard will be released.
\end{abstract}

\section{Introduction}

Entity authentication is a core building block for security in networked systems. In its simplest form, entity authentication boils down to establishing that a party's claimed identity corresponds to its real identity. In practice, stronger guarantees are usually required, such as mutual authentication, agreement among the participating parties on the identities of their peers, or authentication of transmitted data [27,32.

The ISO (International Organization for Standardization) and IEC (International Electrotechnical Commission) jointly provide standards for Information Technology. Their standard 9798 specifies a family of entity authentication protocols. This standard is mandated by numerous other standards that require entity authentication as a building block. Examples include the Guidelines on Algorithms Usage and Key Management [13] by the European Committee for Banking Standards and the ITU-T multimedia standard H.235 [24].

Analysis of previous versions of the ISO/IEC 9798 standard has led to the discovery of several weaknesses $3,8,12$. The standard has been revised several times to address weaknesses and ambiguities, with the latest updates stemming from 2010. One may therefore expect that such a mature and pervasive standard is "bullet-proof" and that the protocols satisfy strong, practically relevant, authentication properties.

On request by CRYPTREC, the Cryptography Research and Evaluation Committee set up by the Japanese Government, we formally analyzed the most recent 
versions of the protocols specified in parts 1-4 of the ISO/IEC 9798 standard using the SCYTHER tool [9]. To our surprise, we not only found that several previously reported weaknesses are still present in the standard, but we also found new weaknesses. In particular, many of the protocols guarantee only weak authentication properties and, under some circumstances, even no authentication at all. For the majority of implementations of the standard where only weak authentication is required, these weaknesses will not lead to security breaches. However, our findings clearly show that the guarantees provided by the protocols are much weaker than might be expected. Moreover, in some cases, additional assumptions are required to ensure even the weakest possible form of authentication.

We analyze the shortcomings in the protocols' design and propose repairs. We justify the correctness of our fixes by providing machine-checked proofs of the repaired protocols. These proofs imply the absence of logical errors: the repaired protocols provide strong authentication properties in a Dolev-Yao model, even when multiple protocols from the standard are run in parallel using the same key infrastructure. Consequently, under the assumption of perfect cryptography, the repaired protocols guarantee strong authentication.

To generate the correctness proofs, we first extend the SCYTHER-PROOF tool [31] to handle bidirectional keys. We then use the tool to generate proof scripts that are checked independently by the Isabelle/HOL theorem prover. As input, SCYTHER-Proof takes a description of a protocol and its properties and produces a proof in higher-order logic of the protocol's correctness. Both the generation of proof scripts and their verification by Isabelle/HOL are completely automatic.

From an engineering perspective, we observe that applying existing principles for constructing cryptographic protocols such as those of Abadi and Needham [2] would not have prevented most of the discovered weaknesses. We therefore additionally propose two design principles in the spirit of [2] whose application would have prevented all of the weaknesses.

Based on our analysis, the ISO/IEC working group responsible for the 9798 standard will release an updated version of the standard, incorporating our proposed fixes.

Summary of Contributions. First, we find previously unreported weaknesses in the most recent version of the ISO/IEC 9798 standard. Second, we repair this practically relevant standard, and provide machine-checked proofs of the correctness of the repairs. Third, we propose two principles for engineering cryptographic protocols in the spirit of [2] that would have prevented the weaknesses. Fourth, our work highlights how modern security protocol analysis tools can be used for falsification and machine-checked verification of security standards.

Organization. In Section 1, we describe the ISO/IEC 9798 standard. In Section2, we model the protocols and analyze them, discovering numerous weaknesses. In Section 3, we analyze the sources of these weaknesses and present two design principles that eliminate them. In Section 4, we explain how we automatically generate machine-checked correctness proofs for these repaired protocols. We describe related work in Section 5 and conclude in Section 6 . 


\section{The ISO/IEC 9798 Standard}

\subsection{Overview}

We give a brief overview of the standard, which specifies a family of entity authentication protocols. We consider here the first four parts of the standard. Part 1 is general and provides background for the other parts. The protocols are divided into three groups. Protocols using symmetric encryption are described in Part 2, those using digital signatures are described in Part 3, and those using cryptographic check functions such as MACs are described in Part 4.

Because the standard has been revised, we also take into account the most recent technical corrigenda and amendments. Our analysis covers the protocols specified by the following documents. For the first part of the standard, we cover ISO/IEC 9798-1:2010 [21]. For the second part, we cover ISO/IEC 97982:2008 18] and Corrigendum 1 from 2010 [22. For the third part, we cover ISO/IEC 9798-3:1998 [16, the corrigendum from 2009 [19, and the amendment from 2010 23. Finally, for the fourth part, our analysis covers ISO/IEC 97984:1999 [17] and the corrigendum from 2009 [20].

Table 1 lists the 17 associated protocols. For each cryptographic mechanism, there are unilateral and bilateral authentication variants. The number of messages and passes differs among the protocols as well as the communication structure. Some of the protocols also use a trusted third party (TTP).

Note that there is no consistent protocol naming scheme shared by the different parts of the ISO/IEC 9798 standard. The symmetric-key based protocols are referred to in [18] as "mechanism 1", "mechanism 2", etc., whereas the protocols in 16, 20, 23. are referred to by their informal name, e.g., "One-pass unilateral authentication". In this paper we will refer to protocols consistently by combining the document identifier, e.g., "9798-2" with a number $n$ to identify the $n$-th protocol in that document. For protocols proposed in an amendment, we continue the numbering from the base document. Hence we refer to the first protocol in [23] as "9798-3-6". The resulting identifiers are listed in Table 1.

Most of the protocols are parameterized by the following elements:

- All text fields included in the protocol specification are optional and their purpose is application-dependent.

- Many fields used to ensure uniqueness or freshness may be implemented either by random numbers, sequence numbers, or timestamps.

- Some protocols specify alternative message contents.

- Some identifier fields may be dropped, depending on implementation details.

\subsection{Notation}

We write $X \| Y$ to denote the concatenation of the bit strings $X$ and $Y$. We write $\{X\}_{k}^{\text {enc }}$ to denote the encryption of $X$ with the symmetric key $k$ and $\{X\}_{k}^{\text {sign }}$ to denote the digital signature of $X$ with the signature key $k$. The application of a cryptographic check function $f$, keyed with key $k$, to a message $m$, is denoted by $f_{k}(m)$. 
Table 1. Protocols specified by Parts $1-4$ of the standard

\begin{tabular}{|c|c|}
\hline Protocol & Description \\
\hline \multicolumn{2}{|c|}{ Part 2: Symmetric-key Cryptography } \\
\hline $9798-2-1$ & One-pass unilateral authentication \\
\hline $9798-2-2$ & Two-pass unilateral authentication \\
\hline $9798-2-3$ & Two-pass mutual authentication \\
\hline $9798-2-4$ & Three-pass mutual authentication \\
\hline $9798-2-5$ & Four-pass with TTP \\
\hline $9798-2-6$ & Five-pass with TTP \\
\hline \multicolumn{2}{|c|}{ Part 3: Digital Signatures } \\
\hline $9798-3-1$ & One-pass unilateral authentication \\
\hline $9798-3-2$ & Two-pass unilateral authentication \\
\hline $9798-3-3$ & Two-pass mutual authentication \\
\hline $9798-3-4$ & Three-pass mutual authentication \\
\hline $9798-3-5$ & Two-pass parallel mutual authentication \\
\hline $9798-3-6$ & Five-pass mutual authentication with TTP, initiated by A \\
\hline $9798-3-7$ & Five-pass mutual authentication with TTP, initiated by B \\
\hline \multicolumn{2}{|c|}{ Part 4: Cryptographic Check Functions } \\
\hline $9798-4-1$ & One-pass unilateral authentication \\
\hline $9798-4-2$ & Two-pass unilateral authentication \\
\hline $9798-4-3$ & Two-pass mutual authentication \\
\hline $9798-4-4$ & Three-pass mutual authentication \\
\hline
\end{tabular}

In the standard, TVP denotes a Time-Variant Parameter, which may be a sequence number, a random value, or a timestamp. $T N$ denotes a time stamp or sequence number. $I_{X}$ denotes the identity of agent $X$. Text $t_{n}$ refers to a text field. These fields are always optional and their use is not specified within the standard. We write $K_{A B}$ to denote the long-term symmetric key shared by $A$ and $B$. If the key is directional, we assume that $A$ uses $K_{A B}$ to communicate with $B$ and that $B$ uses $K_{B A}$. By convention, we use lower case strings for fresh session keys, like $k a b$.

\subsection{Protocol Examples}

Example 1: 9798-4-3. The 9798-4-3 protocol is a two-pass mutual authentication protocol based on cryptographic check functions, e.g., message authentication codes. Its design, depicted in Figure 1 is similar to two related protocols based on symmetric key encryption (9798-2-3) and digital signatures (9798-3-3).

The initiator starts in role $A$ and sends a message that consists of a time stamp or sequence number $T N_{A}$, concatenated with an optional text field and a cryptographic check value. This check value is computed by applying a cryptographic check function to the key shared between $A$ and $B$ and a string consisting of 
Fig. 1. The 9798-4-3 two-pass mutual authentication protocol using a cryptographic check function

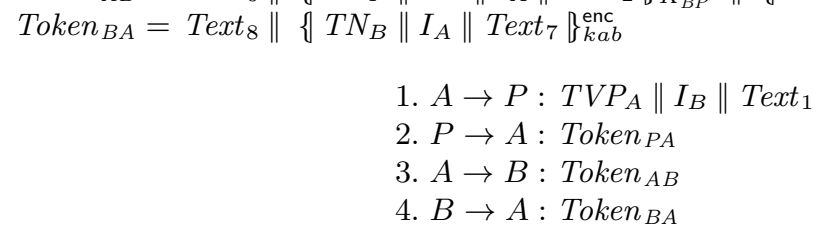

2. $P \rightarrow A:$ Token $_{P A}$

3. $A \rightarrow B$ : Token $_{A B}$

4. $B \rightarrow A:$ Token $_{B A}$

Fig. 2. The 9798-2-5 four pass protocol with TTP using symmetric encryption

$T N_{A}$, B's identity, and optionally a text field $T e x t_{1}$. When $B$ receives this message he computes the cryptographic check himself and compares the result with the received check value. He then computes the response message in a similar way and sends it to $A$, who checks it.

Example 2: 9798-2-5. Figure 2 depicts the 9798-2-5 protocol, which is based on symmetric-key encryption and uses a Trusted Third Party. $A$ first generates a time-variant parameter $T V P_{A}$ (which must be non-repeating), and sends it with $B$ 's identity $I_{B}$ and optionally a text field to the trusted party $P$. $P$ then generates a fresh session key $k a b$ and computes Token $_{P A}$, which essentially consists of two encrypted copies of the key, using the long-term shared keys between $P$ and $A$, and $P$ and $B$, respectively. Upon receiving Token $_{P A}, A$ decrypts the first part to retrieve the session key, and uses the second part to construct Token $A B$. Finally, $B$ retrieves the session key from this message and sends his authentication

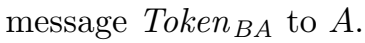

\subsection{Optional Fields and Variants}

There are variants for each protocol listed in Table 1 Each protocol contains text fields, whose purpose is not specified, and which may be omitted, giving rise to another protocol variant. As can be seen in the previous examples, some of these text fields are plaintext, whereas others are within the scope of cryptographic operations (i. e., signed, encrypted, or cryptographically checked). Note that the standard does not provide a rationale for choosing among these options.

In setups where symmetric keys are used, it is common that if Alice wants to communicate with Bob, she will use their shared key, which is the same key that Bob would use to communicate with Alice. Such keys are called bidirectional. Alternatively one can use unidirectional keys where each pair of agents shares two 
symmetric keys, one for each direction. In this case $K_{\text {Alice,Bob }}$ and $K_{\text {Bob,Alice }}$ are different. For some protocols that employ symmetric keys, the standard specifies that if unidirectional keys are used, some identity fields may be omitted from the encrypted (or checked) payload. This yields another variant.

The two protocols 9798-3-6 and 9798-3-7 both provide two options for the tokens included in their messages, giving rise to further variants. Note that in Section 4 we verify corrected versions of all 17 protocols in Table 1, taking all variants into account.

\subsection{Threat Model and Security Properties}

The ISO/IEC 9798 standard neither specifies a threat model nor defines the security properties that the protocols should satisfy. Instead, the introduction of ISO/IEC 9798-1 simply states that the protocols should satisfy mutual or unilateral authentication. Furthermore, the following attacks are mentioned as being relevant: man-in-the-middle attacks, replay attacks, reflection attacks, and forced delay attacks. We note that the standard does not explicitly claim that any of the protocols are resilient against the above attacks.

\section{Protocol Analysis}

We use two different analysis tools. In this section, we use the SCYTHER tool 9] to find attacks on the ISO/IEC 9798 protocols. In Section 4 we will use the related SCYTHER-PROOF tool 31] to generate machine-checked proofs of the corrected versions.

SCYTHER performs an automatic analysis of security protocols in a DolevYao style model, for an unbounded number of instances. It is very efficient at both verification and falsification, in particular for authentication protocols such as those considered here. Using SCYTHER, we performed protocol analysis with respect to different forms of authentication. We explain these forms below when discussing particular protocols.

Our analysis reveals that the majority of the protocols in the standard ensure weak entity authentication. However, we also found attacks on five protocols and two protocol variants. These attacks fall into the following categories: role-mixup attacks, type flaw attacks, multiple-role TTP attacks, and reflection attacks. In all cases, when an agent finishes his role of the protocol, the protocol has not been executed as expected, which can lead the agent to proceed on false assumptions about the state of the other involved agents.

In Table 2 we list the attacks we found using Scyther. The rows list the protocols, the properties violated, and any additional assumptions required for the attacks. We have omitted in the table all attacks that are necessarily entailed by the attacks listed. For example, since 9798-2-5 does not satisfy aliveness from $B$ 's perspective, it also does not satisfy any stronger properties such as (weak) agreement. We now describe the classes of attacks in more detail. 
Table 2. Overview of attacks found

\begin{tabular}{lll}
\hline Protocol & Violated property & Assumptions \\
\hline $9798-2-3$ & A Agreement(B,TNB,Text3) & \\
$9798-2-3$ & B Agreement(A,TNA,Text1) & \\
$9798-2-3-$ udkey & A Agreement(B,TNB,Text3) & \\
$9798-2-3-$ udkey & B Agreement(A,TNA,Text1) & Alice-talks-to-Alice \\
$9798-2-5$ & A Alive & \\
$9798-2-5$ & B Alive & \\
$9798-2-6$ & A Alive & \\
$9798-2-6$ & B Alive & \\
$9798-3-3$ & A Agreement(B,TNB,Text3) & \\
$9798-3-3$ & B Agreement(A,TNA,Text1) & Type-flaw \\
$9798-3-7-1$ & A Agreement(B,Ra,Rb,Text8) & \\
$9798-4-3$ & A Agreement(B,TNb,Text3) & \\
$9798-4-3$ & B Agreement(A,TNa,Text1) & \\
$9798-4-3-$ udkey & A Agreement(B,TNb,Text3) & \\
$9798-4-3-$ udkey & B Agreement(A,TNa,Text1) & \\
\hline
\end{tabular}

\subsection{Role-Mixup Attacks}

Some protocols are vulnerable to a role-mixup attack in which an agent's assumptions on another agent's role are wrong. Many relevant forms of strong authentication, such as agreement [27, matching conversations [4] or synchronisation [10, require that when Alice finishes her role apparently with Bob, then Alice and Bob not only agree on the exchanged data, but additionally Alice can be sure that Bob was performing in the intended role. Protocols vulnerable to role-mixup attacks violate these strong authentication properties.

Figure 3 on the following page shows an example of a role-mixup attack on the 9798-4-3 protocol from Figure 1. Agents perform actions such as sending and receiving messages, resulting in message transmissions represented by horizontal arrows. Actions are executed within threads, represented by vertical lines. The box at the top of each thread denotes the parameters involved in the thread's creation. Claims of security properties are denoted by hexagons and a crossed-out hexagon denotes that the claimed property is violated.

In this attack, the adversary uses a message from Alice in role A (thread 1) to trick Alice in role B (thread 3) into thinking that Bob is executing role A and is trying to initiate a session with her. However, Bob (thread 2) is only replying to a message provided to him by the adversary, and is executing role $\mathrm{B}$. The adversary thereby tricks Alice into thinking that Bob is in a different state than he actually is.

Additionally, when the optional text fields Text $_{1}$ and Text $_{3}$ are used, the rolemixup attack also violates the agreement property with respect to these fields: Alice will end the protocol believing that the optional field data she receives from Bob was intended as Text1, whereas Bob actually sent this data in the 


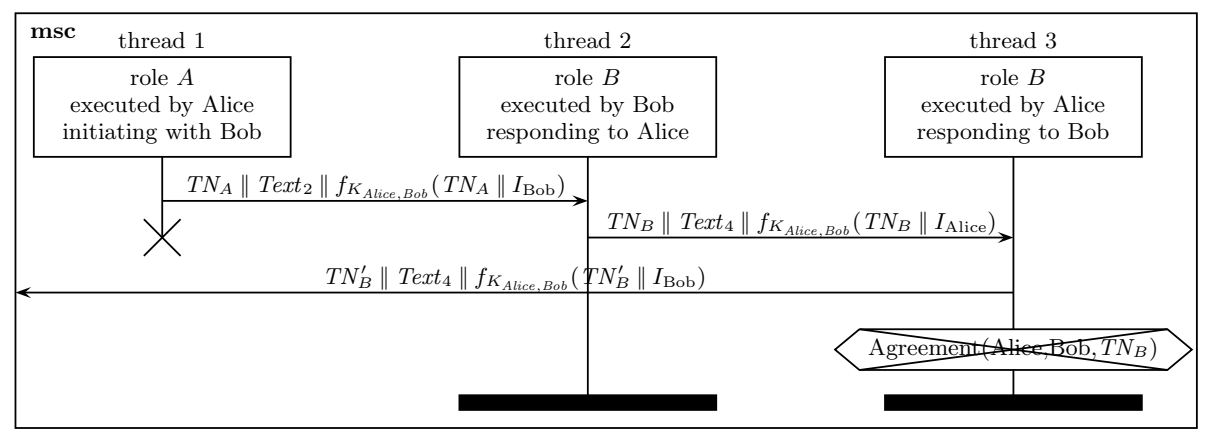

Fig. 3. Role-mixup attack on 9798-4-3: when Alice finishes thread 3 she wrongly assumes that Bob was performing the $A$ role

Text3 field. Depending on the use of these fields, this can constitute a serious security problem. Note that exploiting these attacks, as well as the other attacks described below, does not require "breaking" cryptography. Rather, the adversary exploits similarities among messages and the willingness of agents to engage in the protocol.

Summarizing, we found role-mixup attacks on the following protocols: 97982-3 with bi- or unidirectional keys, 9798-2-5, 9798-3-3, and 9798-4-3 with bi- or unidirectional keys.

\subsection{Type Flaw Attacks}

Some protocol implementations are vulnerable to type flaw attacks where data of one type is parsed as data of another type. Consider, for example, an implementation where agent names are encoded into bit-fields of length $n$, which is also the length of the bit-fields representing nonces. It may then happen that an agent who expects to receive a nonce (any fresh random value that it has not seen before), therefore accepts a bit string that was intended to represent an agent name.

SCYTHER finds such an attack on the 9798-3-7 protocol, also referred to as "Five pass authentication (initiated by B)" [23, p. 4]. In the attack, both (agent) Alice and (trusted party) Terence mistakenly accept the bit string corresponding to the agent name "Alice" as a nonce.

\subsection{Attacks Involving TTPs That Perform Multiple Roles}

Another class of attacks occurs when parties can perform both the role of the trusted third party and another role. This scenario is not currently excluded by the standard.

In Figure 4 we show an attack on 9798-2-5, from Figure 2 The attack closely follows a regular protocol execution. In particular, threads 1 and 3 perform the 


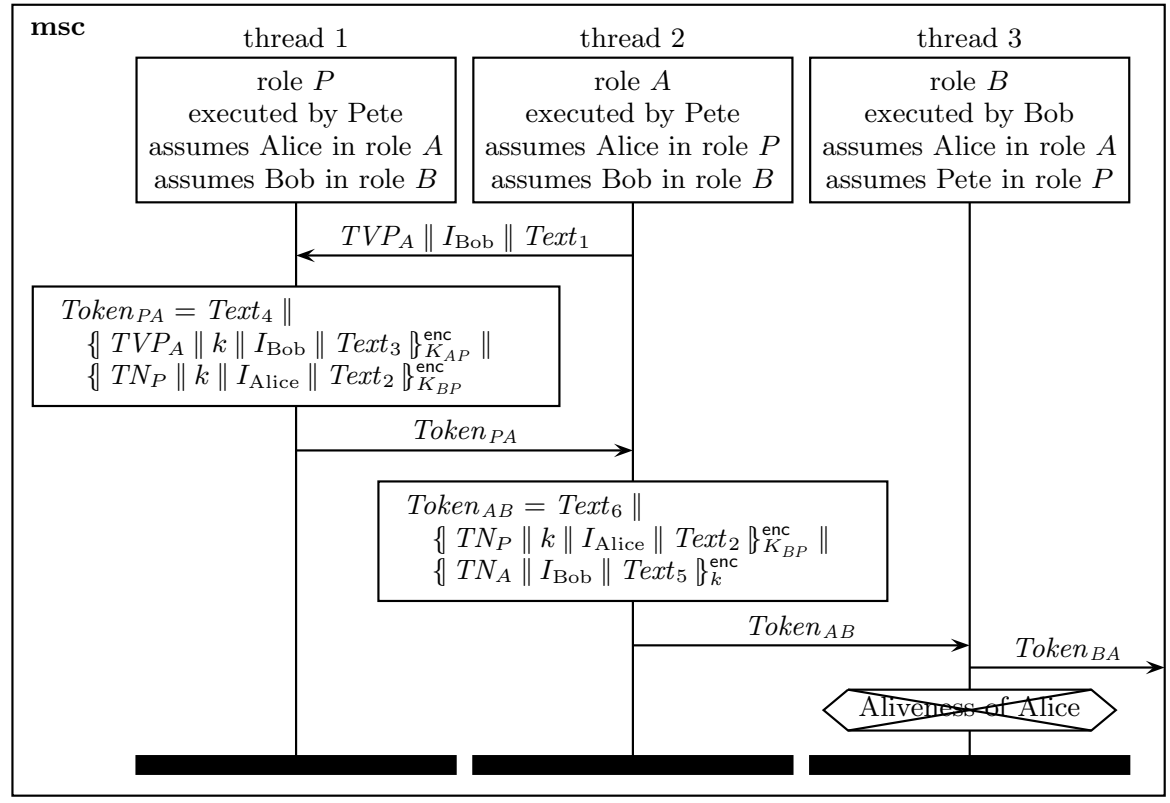

Fig. 4. Attack on the 9798-2-5 protocol where the trusted third party Pete performs both the $P$ role and the $A$ role. The assumptions of thread 1 and 3 agree. Bob wrongly concludes that Alice is alive.

protocol as expected. The problem is thread 2. Threads 1 and 3 assume that the participating agents are Alice (in the $A$ role), Bob (in the $B$ role), and Pete (in the $P$ role). From their point of view, Alice should be executing thread 2 . Instead, thread 2 is executed by Pete, under the assumption that Alice is performing the $P$ role. Thread 2 receives only a single message in the attack, which is Token $P A$. Because the long-term keys are symmetric, thread 2 cannot determine from the part of the message encrypted with $K_{A P}$ that thread 1 has different assumptions. Thread 2 just forwards the other encrypted message part blindly to thread 3 , as it does not expect to be able to decrypt it. Finally, thread 3 cannot detect the confusion between Alice and Pete, because the information in Token AB $_{B}$ that was added by thread 2 only includes Bob's name.

Summarizing, we found attacks involving TTPs that perform multiple roles on the 9798-2-5 and 9798-2-6 protocol.

\subsection{Reflection Attacks}

Reflection attacks occur when agents may start sessions communicating with the same identity, a so-called Alice-talks-to-Alice scenario. The feasibility and relevance of this scenario depends on the application and its internal checks.

If an Alice-talks-to-Alice scenario is possible, some protocols are vulnerable to reflection attacks. The Message Sequence Chart in Figure 5 shows an example for 


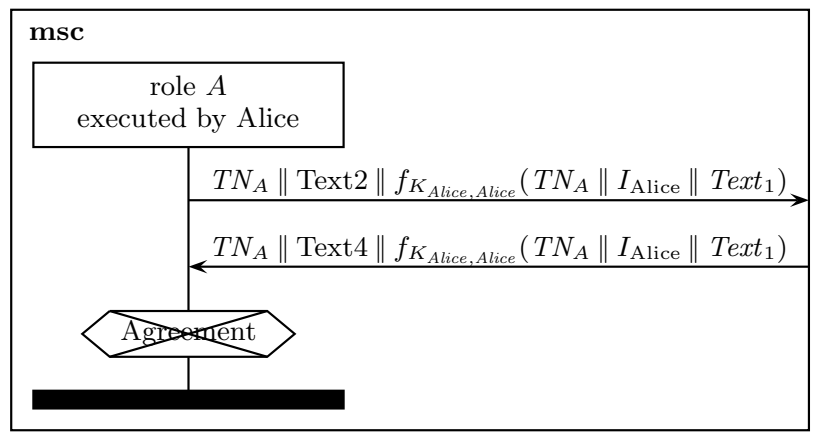

Fig. 5. Reflection attack on 9798-4-3

the 9798-4-3 protocol from Figure 1 In this attack, the adversary (not depicted) reflects the time stamp (or nonce) and cryptographic check value from the message sent by Alice back to the same thread, while prepending the message Text4. This attack violates one of the main requirements explicitly stated in the ISO/IEC 97981 introduction, namely absence of reflection attacks.

Summarizing, we found reflection attacks on the following protocols: 9798-23 with bi- or unidirectional keys, 9798-2-5, 9798-3-3, and 9798-4-3 with bi- or unidirectional keys.

\section{Repairing the Protocols}

\subsection{Root Causes of the Problems}

We identify two shortcomings in the design of the protocols, which together account for all of the weaknesses detected.

1) Cryptographic Message Elements May Be Accepted at Wrong Positions. In both the reflection and role mixup attacks, the messages that are received in a particular step of a role were not intended to be received at that position. By design, the protocol messages are all similar in structure, making it impossible to determine at which point in the protocols the messages were intended to be received.

As a concrete example, consider the reflection attack in Figure 5. Here, the message sent in the protocol's first step can be accepted in the second step, even though this is not part of the intended message flow.

2) Underspecification of the Involved Identities and their Roles. As noted, the symmetric-key based protocols with a TTP, 9798-2-5 and 9798-2-6, do not explicitly state that entities performing the TTP role cannot perform other roles. Hence it is consistent with the standard for Alice to perform both the role of the TTP as well as role A or B. In these cases, the aliveness of the partner cannot 
be guaranteed, as explained in Section 2.3. The source of this problem is that one cannot infer from each message which identity is associated to which role.

For example, consider the first encrypted component from the third message in the 9798-2-5 protocol with bidirectional keys, in Figure 2.

$$
\left\{T N_{P}\|k a b\| I_{A} \| \text { Text }_{2}\right\}_{K_{B P}}^{\mathrm{enc}}
$$

This message implicitly includes the identities of the three involved agents: the identity of the agent performing the $A$ role is explicitly included in the encryption, and the shared long-term key $K_{B P}$ implicitly associates the message to the key shared between the agent performing the $B$ and $P$ roles. However, because the key is bidirectional, the recipient cannot determine which of the two agents (say, Bob and Pete) sharing the key performed which role: either Bob performed the $B$ role and Pete the $P$ role, or vice versa. Our attack exploits exactly this ambiguity.

\subsection{Associated Design Principles}

To remedy these problems, we propose two principles for designing security protocols. These principles are in the spirit of Abadi and Needham's eleven principles for prudent engineering practice for cryptographic protocols [2].

Our first principle concerns tagging.

Principle: positional tagging. Cryptographic message components should contain information that uniquely identifies their origin. In particular, the information should identify the protocol, the protocol variant, the message number, and the particular position within the message, from which the component was sent.

This is similar in spirit to Abadi and Needham's Principle 1, which states that "Every message should say what it means: the interpretation of the message should depend only on its content. It should be possible to write down a straightforward English sentence describing the content - though if there is a suitable formalism available that is good too." Our principle does not depend on the meaning of the message as intended by the protocol's designer. Instead, it is based solely on the structure of the protocol messages and their acceptance conditions.

Note that we consider protocols with optional fields to consist of multiple protocol variants. Thus, a message component where fields are omitted, should contain information to uniquely determine which fields were omitted.

Our second principle is a stricter version of Abadi and Needham's Principle 3.

Principle: inclusion of identities and their roles. Each cryptographic message component should include information about the identities of all the agents involved in the protocol run and their roles, unless there is a compelling reason to do otherwise.

A compelling reason to leave out identity information might be that identity hiding is a requirement, i. e., Alice wants to hide that she is communicating with 
Bob. However, such requirements can usually be met by suitably encrypting identity information.

Contrast this principle with the Abadi and Needham's Principle 3: "If the identity of a principal is essential to the meaning of a message, it is prudent to mention the principal's name explicitly in the message." The original principle is only invoked when the identity is essential. Instead, we propose to always include information on all the identities as well as their roles. This principle would have prevented attacks on many protocols, including the attacks on the 9798-2-5 and 9798-2-6 protocols, as well as the Needham-Schroeder protocol [26].

For protocols with a fixed number of roles, this principle can be implemented by including an ordered sequence of the identities involved in each cryptographic message component, such that the role of an agent can be inferred from its position in the sequence.

\subsection{Proposed Modifications to the Standard}

All the previously mentioned attacks on the ISO/IEC 9798 can be prevented by applying the previous two principles. Specifically, we propose three modifications to the ISO standard, shown in Figure 6. The first two directly follow from the principles and the third modification restricts the use of two protocols in the standard. Afterwards we give an example of a repaired protocol.

Note that in this section we only give informal arguments why our modifications prevent the attacks. In Section 4, we provide machine-checked proofs that this is the case.

\section{Ensuring That Cryptographic Data Cannot Be Accepted at the Wrong}

Point. We factor the first principle (positional tagging) into two parts and propose two corresponding amendments to the standard. First, we explicitly include in each cryptographic message component constants that uniquely identify the protocol, the message number, and the position within the message. Second, we ensure that protocol variants can be uniquely determined from the messages.

In our first amendment, shown in Figure 6, we implement unique protocol identifiers by using an existing part of the standard: the object identifier from Annex B of the standard, which specifies an encoding of a unique protocol identifier. We also introduce a unique identifier for the position of the cryptographic component within the protocol.

Amendment 1 prevents all reflection attacks because messages sent in one step will no longer be accepted in another step. Furthermore, it prevents all role mixup attacks, because the unique constants in the messages uniquely determine the sending role. The final part of Amendment 1, stating that cryptographic keys should not be used by other protocols, provides distinctness of cryptographic messages with respect to any other protocols.

Our second amendment, also shown in Figure 6, ensures that the protocol variant (determined by the omission of optional fields) can be uniquely determined from the messages. We implement this by requiring that the recipient of a message can uniquely determine which optional fields, if any, were omitted. 


\section{Amendment 1:}

The cryptographic data (encryptions, signatures, cryptographic check values) used at different places in the protocols must not be interchangeable. This may be enforced by including in each encryption/signature/CCF value the following two elements:

1. The object identifier as specified in Annex B [23, p. 6], in particular identifying the ISO standard, the part number, and the authentication mechanism.

2. For protocols that contain more than one cryptographic data element, each encryption must contain a constant that uniquely identifies the position of the element within the protocol.

The recipient of a message must verify that the object identifier and the position identifiers are as expected. The cryptographic keys used by implementations of the ISO/IEC 9798 protocols must be distinct from the keys used by other protocols.

\section{Amendment 2:}

When optional fields, such as optional identities or optional text fields, are not used then they must be set to empty. In particular, the message encoding must ensure that the concatenation of a field and an empty optional field is uniquely parsed as a concatenation. This can be achieved by implementing optional fields as variable-length fields. If the optional field is not used, the length of the field is set to zero.

\section{Amendment 3:}

Entities that perform the role of the TTP in the 9798-2-5 and 9798-2-6 protocols must not perform the A or B role.

Fig. 6. Proposed amendments to the ISO/IEC 9798 standard

To see why protocols with omitted optional fields must be considered as protocol variants, consider the following example: Consider a protocol in which a message contains the sequence $X\left\|I_{A}\right\|$ Text, where $I_{A}$ is an identity field that may be dropped (e.g., with unidirectional keys) and Text is an optional text field. Then, it may be the case that in one protocol variant, an agent expects a message of the form $X \| I_{A}$, whereas the other implementation expects a message of the form $X \|$ Text. The interaction between the two interpretations can result in attacks. For example, the text field is used to insert a false agent identity, or an agent identity is wrongly assumed to be the content of the text field.

If we follow the second amendment in the above example, the expected messages correspond to $X\left\|I_{A}\right\| \perp$ and $X\|\perp\|$ Text, respectively, where $\perp$ denotes the zero-length field. Because the ISO/IEC 9798 standard requires that concatenated fields can be uniquely decomposed into their constituent parts, misinterpretation of the fields is no longer possible.

Together, Amendments 1 and 2 implement our first principle.

Addressing Underspecification of the Role Played by Agents. Almost all the protocols in the ISO/IEC 9798 standard already adhere to our second principle: unique identification of the involved parties and their roles. However, all protocols in the standard conform to Abadi and Needham's third principle because the messages uniquely determine the identities of all involved parties. 
1. $A \rightarrow B: T N_{A}\left\|T e x t_{2}\right\| f_{K_{A B}}\left(\right.$ "9798-4-3 ccf1" $\left.\left\|T N_{A}\right\| I_{B} \| \perp\right)$

2. $B \rightarrow A: T N_{B}\left\|\operatorname{Text}_{4}\right\| f_{K_{A B}}\left(\right.$ “9798-4-3 ccf2" $\left\|T N_{B}\right\| I_{A} \|$ Text $\left._{3}\right)$

Fig. 7. Repaired version of the 9798-4-3 protocol with omitted Text ${ }_{1}$ field

There are only two protocols in the standard that conform to Abadi and Needham's principle but not to our second principle: 9798-2-5 and 9798-2-6. For example, the messages of the 9798-2-5 protocol identify all parties involved by their association to the long-term keys. However they do not conform to our second principle because the roles of the involved identities cannot be uniquely determined from the messages. This is the underlying reason why, as currently specified, the 9798-2-5 and 9798-2-6 protocols do not guarantee the aliveness of the partner, as shown in Section 2.3 .

This problem can be solved by applying our principle, i. e., including the identities of all three participants in each message, so that their roles can be uniquely determined. This is an acceptable solution and we have formally verified it using the method of Section 4. However, from our analysis with SCYTHER, we observe that the attacks require that the Trusted Third Party also performs other roles (A or B). Under the assumption that in actual applications a TTP will, by definition, not perform the $\mathrm{A}$ or $\mathrm{B}$ role, the protocols already provide strong authentication. Thus, an alternative solution is to leave the protocols unchanged and make this restriction explicit. This results in more streamlined protocols and also requires minimal changes to the standard. This is the proposal made in Amendment 3 in Figure 6. We have also verified this solution as described in Section 4.

Repaired Protocols. Applying our principles and proposed amendments to the standard, we obtain repaired versions of the protocols. As an example, we show the repaired version of the 9798-4-3 protocol with bidirectional keys in Figure [7. In this example, the Text 1 field is not used, and is therefore replaced by $\perp$. Each use of a cryptographic primitive (in this case the cryptographic check function) includes a constant that uniquely identifies the protocol (9798-4-3) as well as the position within the protocol specification ( $\operatorname{cff} 1$ and $\operatorname{ccf} 2$ ).

\section{Proving the Correctness of the Repaired Protocols}

The principles and amendments proposed in the previous section are motivated by our analysis of the attacks and the protocol features that enable them. Consequently, the principles and amendments are designed to eliminate these undesired behaviors. Such principles are useful guides for protocol designers but their application does not strictly provide any security guarantees. In order to ensure that the repaired protocols actually have the intended strong authentication properties, we provide machine-checked correctness proofs.

We use a version of the SCYTHER-PROOF tool proposed in 31] to generate proofs of these properties. Given a description of a protocol and its security 
properties, the tool generates a proof script that is afterwards automatically checked by the Isabelle/HOL theorem prover [33. If the prover succeeds, then the protocol is verified with respect to a symbolic, Dolev-Yao model. To verify our repaired protocols, we extended the tool with support for bidirectional symmetric long-term keys.

The proofs generated by SCYTHER-PROOF are based on a security protocol verification theory that provides a sound way to perform finite case distinctions on the possible sources of messages that are known to the intruder, in the context of a given protocol. The generated proofs use these case distinctions to show that the assumptions of a security property imply its conclusions, as explained in Example 1. The theorems constituting this verification theory are formally derived in Isabelle/HOL from the formalization of a symbolic, Dolev-Yao model.

The tool searches for the proofs with the fewest number of such case distinctions. For example, in the proofs of our repaired protocols, two such case distinctions are required on average to prove a security property. Therefore, the generated proof scripts (available from [1]) are amenable to human inspection and understanding. To simplify the task of understanding how the proofs work and, hence, why the protocol is correct, the tool also generates proof outlines. These consist of a representation of the security property proven and a tree of case distinctions constituting the proof.

For each repaired protocol, we prove that it satisfies non-injective agreement on all data items within the scope of cryptographic operators in the presence of a Dolev-Yao intruder. Moreover, we prove that this holds even when all the protocols from the standard are executed in parallel using the same key infrastructure, provided that the set of bidirectional keys is disjoint from the set of unidirectional keys. As the content of text fields is underspecified in the standard, we assume that the intruder chooses their content immediately before they are sent. We model timestamps and sequence numbers by random numbers that are chosen at the beginning of a role and are public.

Example 1. Figure 8 on the next page specifies our model of the repaired 9798-4-3 protocol with bidirectional keys in the input language of the SCYTHER-PROOF tool. The leak_A and the leak_B steps model that the timestamps (represented here as randomly generated numbers) are publicly known by leaking them to the intruder. We model that the contents of the text_1 and text_2 fields is chosen by the intruder by defining them as variables that receive their content from the network, and therefore from the intruder. We model the cryptographic check function by the hash function $h$.

Figure 9 on the following page shows the proof outline for non-injective agreement for the A-role of this protocol, which is automatically generated by our tool. We have taken minor liberties here in its presentation to improve readability. In the figure, \#i is a symbolic variable denoting some thread $i$ and A\# $i$ is the value of the A-variable in the thread $i$. Lines 3-9 state the security property: for all threads \#i that execute the A-role and have executed its Step 2 with uncompromised (honest) agents $A \# i$ and $B \# i$, there exists some thread $\# j$ that 


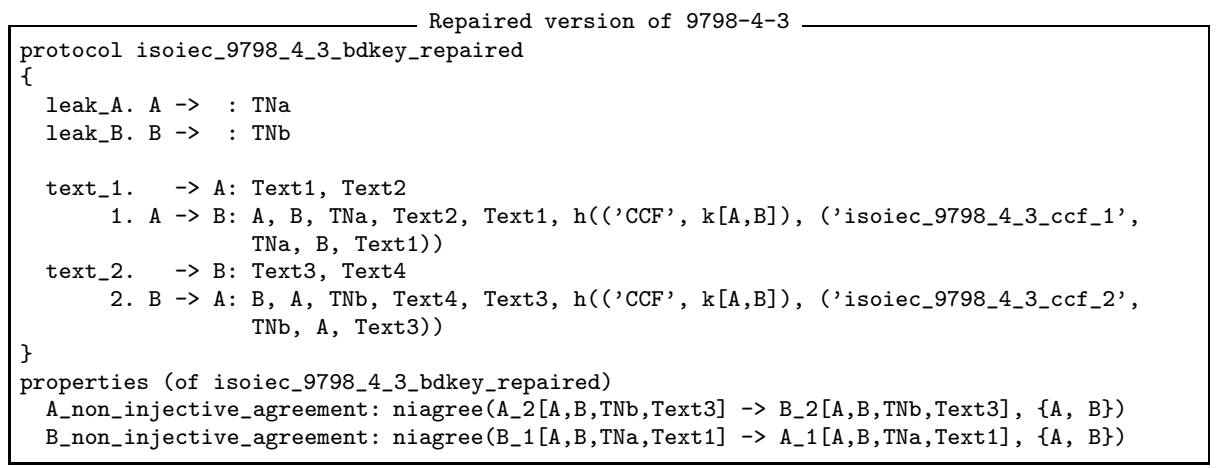

Fig. 8. Example input provided to the SCYTHER-Proof tool

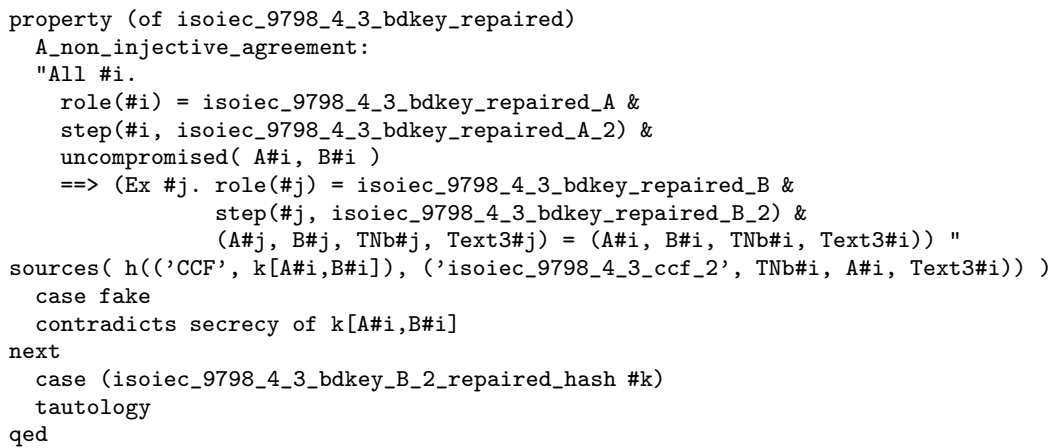

Fig. 9. Example proof outline automatically produced by the SCYTHER-PROOF tool

executed Step 2 of the B-role and thread \# j agrees with thread \#i on the values of $\mathrm{A}, \mathrm{B}, \mathrm{TNb}$, and Text3.

The proof proceeds by observing that thread \#i executed Step 2 of the Arole. Therefore, thread \#i received the hash in line 10 from the network, which implies that the intruder knows this hash. For our protocol, a case distinction on the sources of this hash results in two cases: (1) the intruder could have constructed (faked) this hash by himself or (2) the intruder could have learned this hash from some thread \#k that sent it in Step 2 of the B-role. There are no other cases because all other hashes have different tags. Case 1 is contradictory because the intruder does not know the long-term key shared between the two uncompromised agents $\mathrm{A \#} i$ and $\mathrm{B \# i}$. In Case 2, the security property holds because we can instantiate thread \# $j$ in the conclusion with thread \#k. Thread \#k executed Step 2 of the B-role and agrees with thread \#i on all desired values because they are included in the hash. 
We verify the parallel composition of all repaired protocols of the ISO/IEC 9798 standard as follows. Given the disjoint encryption theorem [14, it is sufficient to verify only the parallel composition of protocols that use the same cryptographic primitive and the same keys. We verify the properties of each protocol when composed in parallel with all other protocols that use the same cryptographic primitive and the same keys. Note that in the corresponding proofs, the case distinctions on the source of messages known to the intruder range over the roles of each protocol in the protocol group. Despite the substantial increase in the scope of these case distinctions, the proof structure of the composed protocols is the same as for the individual protocols, as all additional cases are always trivially discharged due to the tagging: cryptographic components received by a thread of one protocol contain tags that do not match with the tags in messages produced by roles from other protocols.

Our extension of the SCYTHER-PROOF tool as well as the protocol models (including the property specifications) can be downloaded at [1]. Using a Core 2 Duo $2.20 \mathrm{GHz}$ laptop with 2GB RAM, the full proof script generation requires less than 20 seconds, and Isabelle's proof checking requires less than three hours.

\section{Related Work}

Previous Analyses of the ISO/IEC 9798 Protocols. Chen and Mitchell [8] reported attacks based on parsing ambiguities on protocols from several standards. They identify two types of ambiguities in parsing strings involving concatenation: (1) recipients wrongly parse an encrypted string after decryption, or (2) recipients wrongly assume that a different combination of data fields was input to the digital signature or MAC that they are verifying. They show that such errors lead to attacks, and propose modifications to the standards. Their analysis resulted in a technical corrigendum to the ISO/IEC 9798 standard [19,20,22.

Some of the protocols have been used as case studies for security protocol analysis tools. In [12, the Casper/FDR tool is used to discover weaknesses in six protocols from the ISO/IEC 9798 standard. The attacks discovered are similar to our reflection and role-mixup attacks. They additionally report so-called multiplicity attacks, but these are prevented by following the specification of the time-variant parameters in Part 1 of the standard. Contrary to our findings, their analysis reports "no attack" on the 9798-2-5 and 9798-2-6 protocols as they do not consider type-flaw attacks. A role-mixup attack on the $9798-3-3$ protocol was also discovered by the SATMC tool [3]. Neither of these two works suggested how to eliminate the detected weaknesses.

In [11, the authors verify the three-pass mutual authentication protocols that use symmetric encryption and digital signatures, i. e., 9798-2-4 and 9798-3-4. Their findings are consistent with our results.

Related Protocols. The SASL authentication mechanism from RFC 3163 [34] claims to be based on Part 3 of the ISO/IEC 9798 standard. However, the SASL protocol is designed differently than the ISO/IEC protocols and is vulnerable to 
a man-in-the-middle attack similar to Lowe's well-known attack on the NeedhamSchroeder public-key protocol. Currently, the SASL protocol is not recommended for use (as noted in the RFC). The SASL protocol only provides authentication in the presence of an eavesdropping adversary, which can also be achieved using only plaintext messages.

In the academic literature on key exchange protocols, one finds references to a Diffie-Hellman-based key exchange protocol known as "ISO 9798-3". This protocol seems to be due to [7, p. 464-465], where a protocol is given that is similar in structure to the three-pass mutual authentication ISO/IEC 9798 protocol based on digital signatures, where each random value $n$ is replaced by ephemeral public keys of the form $g^{x}$. However, in the actual ISO/IEC 9798 standard, no key exchange protocols are defined, and no protocols use Diffie-Hellman exponentiation.

\section{Conclusions}

Our findings show that great care must be taken when using current implementations of the ISO/IEC 9798 standard. Under the assumption that trusted third parties do not play other roles, the protocols guarantee a weak form of authentication, namely, aliveness. However, many of the protocols do not satisfy any stronger authentication properties, which are needed in realistic applications. For example, when using these protocols one cannot assume that when a text field is encrypted with a key and was apparently sent by Bob, that Bob indeed sent it, or that he was performing the intended role. In contrast, our repaired versions satisfy strong authentication properties and hence ensure not only aliveness but also agreement on the participating agents, their roles, the values of time-variant parameters, and the message fields that are cryptographically protected.

Based on our analysis of the standard's weaknesses, we have proposed amendments and provided machine-checked proofs of their correctness. Our proofs guarantee the absence of these weaknesses even in the case that all protocols from the standard are run in parallel using the same key infrastructure. The working group responsible for the ISO/IEC 9798 standard will release an updated version of the standard based on our analysis and proposed fixes.

Formal methods are slowly starting to have an impact in standardization bodies, e.g., [5, 6, 15, 25, 29, 30. We expect this trend to continue as governments and other organizations increasingly push for the use of formal methods for the development and evaluation of critical standards. For example, ISO/IEC JTC 1/SC 27 started the project "Verification of cryptographic protocols (ISO/IEC 29128)" in 2007 which is developing standards for certifying cryptographic protocols, where the highest evaluation levels require the use of formal, machine checked correctness proofs [28].

We believe that the approach we have taken here to analyze and provably repair the ISO/IEC 9798 standard can play an important role in future standardization efforts. Our approach supports standardization committees with both falsification, for analysis in the early phases of standardization, and verification, providing objective and verifiable security guarantees in the end phases. 


\section{References}

1. Models and proofs of the repaired ISO/IEC 9798 protocols and source code of Scyther-Proof (May 2011), http://www.infsec.ethz.ch/research/software\#ESPL

2. Abadi, M., Needham, R.: Prudent engineering practice for cryptographic protocols. IEEE Transactions on Software Engineering 22(1), 6-15 (1996)

3. Armando, A., Compagna, L.: SAT-based model-checking for security protocols analysis. Int. J. Inf. Sec. 7(1), 3-32 (2008)

4. Bellare, M., Rogaway, P.: Entity Authentication and Key Distribution. In: Stinson, D.R. (ed.) CRYPTO 1993. LNCS, vol. 773, pp. 232-249. Springer, Heidelberg (1994)

5. Bhargavan, K., Fournet, C., Corin, R., Zalinescu, E.: Cryptographically verified implementations for TLS. In: ACM Conference on Computer and Communications Security, pp. 459-468. ACM (2008)

6. Bhargavan, K., Fournet, C., Gordon, A.D., Swamy, N.: Verified implementations of the information card federated identity-management protocol. In: ASIACCS, pp. 123-135. ACM (2008)

7. Canetti, R., Krawczyk, H.: Analysis of Key-Exchange Protocols and Their Use for Building Secure Channels. In: Pfitzmann, B. (ed.) EUROCRYPT 2001. LNCS, vol. 2045, pp. 453-474. Springer, Heidelberg (2001)

8. Chen, L., Mitchell, C.J.: Parsing ambiguities in authentication and key establishment protocols. Int. J. Electron. Secur. Digit. Forensic 3, 82-94 (2010)

9. Cremers, C.J.F.: The Scyther Tool: Verification, Falsification, and Analysis of Security Protocols. In: Gupta, A., Malik, S. (eds.) CAV 2008. LNCS, vol. 5123, pp. 414-418. Springer, Heidelberg (2008), http://people.inf.ethz.ch/cremersc/scyther/

10. Cremers, C., Mauw, S., de Vink, E.: Injective synchronisation: an extension of the authentication hierarchy. Theoretical Computer Science, 139-161 (2006)

11. Datta, A., Derek, A., Mitchell, J., Pavlovic, D.: Abstraction and refinement in protocol derivation. In: Proc. 17th IEEE Computer Security Foundations Workshop (CSFW), pp. 30-45. IEEE Comp. Soc. (June 2004)

12. Donovan, B., Norris, P., Lowe, G.: Analyzing a library of security protocols using Casper and FDR. In: Proc. of the Workshop on Formal Methods and Security Protocols (1999)

13. European Payments Council. Guidelines on algorithms usage and key management. Technical report, EPC342-08 Version 1.1 (2009)

14. Guttman, J.D., Thayer, F.J.: Protocol independence through disjoint encryption. In: CSFW, pp. 24-34 (2000)

15. He, C., Sundararajan, M., Datta, A., Derek, A., Mitchell, J.C.: A modular correctness proof of IEEE 802.11i and TLS. In: Proc. of the 12th ACM Conference on Computer and Communications Security, CCS 2005, pp. 2-15. ACM, New York (2005)

16. International Organization for Standardization, Genève, Switzerland. ISO/IEC 9798-3:1998, Information technology - Security techniques - Entity Authentication - Part 3: Mechanisms using digital signature techniques, 2nd edn. (1998)

17. International Organization for Standardization, Genève, Switzerland. ISO/IEC 9798-4:1999, Information technology - Security techniques - Entity Authentication - Part 3: Mechanisms using a cryptographic check function, 2nd edn. (1999) 
18. International Organization for Standardization, Genève, Switzerland. ISO/IEC 9798-2:2008, Information technology - Security techniques - Entity Authentication - Part 2: Mechanisms using symmetric encipherment algorithms, 3rd edn. (2008)

19. International Organization for Standardization, Genève, Switzerland. ISO/IEC 9798-3:1998/Cor.1:2009, Information technology - Security techniques - Entity Authentication - Part 3: Mechanisms using digital signature techniques. Technical Corrigendum 1 (2009)

20. International Organization for Standardization, Genève, Switzerland. ISO/IEC 9798-4:1999/Cor.1:2009, Information technology - Security techniques - Entity Authentication - Part 3: Mechanisms using a cryptographic check function. Technical Corrigendum 1 (2009)

21. International Organization for Standardization, Genève, Switzerland. ISO/IEC 9798-1:2010, Information technology - Security techniques - Entity Authentication - Part 1: General, 3rd edn. (2010)

22. International Organization for Standardization, Genève, Switzerland. ISO/IEC 9798-2:2008/Cor.1:2010, Information technology - Security techniques - Entity Authentication - Part 2: Mechanisms using symmetric encipherment algorithms. Technical Corrigendum 1 (2010)

23. International Organization for Standardization, Genève, Switzerland. ISO/IEC 9798-3:1998/Amd.1:2010, Information technology - Security techniques - Entity Authentication - Part 3: Mechanisms using digital signature techniques. Amendment 1 (2010)

24. ITU-T. Recommendation H.235 - Security and encryption for H-series (H.323 and other H.245-based) multimedia terminals (2003)

25. Kuhlman, D., Moriarty, R., Braskich, T., Emeott, S., Tripunitara, M.: A correctness proof of a mesh security architecture. In: Proc. of the 2008 21st IEEE Computer Security Foundations Symposium, pp. 315-330. IEEE Computer Society (2008)

26. Lowe, G.: Breaking and Fixing the Needham-Schroeder Public-Key Protocol Using FDR. In: Margaria, T., Steffen, B. (eds.) TACAS 1996. LNCS, vol. 1055, pp. 147166. Springer, Heidelberg (1996)

27. Lowe, G.: A hierarchy of authentication specifications. In: Proc. 10th IEEE Computer Security Foundations Workshop (CSFW), pp. 31-44. IEEE (1997)

28. Matsuo, S., Miyazaki, K., Otsuka, A., Basin, D.: How to Evaluate the Security of Real-Life Cryptographic Protocols? - the Cases of ISO/IEC 29128 and CRYPTREC. In: Sion, R., Curtmola, R., Dietrich, S., Kiayias, A., Miret, J.M., Sako, K., Sebé, F. (eds.) FC 2010 Workshops. LNCS, vol. 6054, pp. 182-194. Springer, Heidelberg (2010)

29. Meadows, C.: Analysis of the Internet Key Exchange protocol using the NRL Protocol Analyzer. In: IEEE Symposium on Security and Privacy, pp. 216-231 (1999)

30. Meadows, C., Syverson, P.F., Cervesato, I.: Formal specification and analysis of the Group Domain Of Interpretation Protocol using NPATRL and the NRL Protocol Analyzer. Journal of Computer Security 12(6), 893-931 (2004)

31. Meier, S., Cremers, C.J.F., Basin, D.A.: Strong invariants for the efficient construction of machine-checked protocol security proofs. In: CSF, pp. 231-245. IEEE Computer Society (2010)

32. Menezes, A., Oorschot, P.V., Vanstone, S.: Handbook of Applied Cryptography, 5th edn. CRC Press, Inc. (2001)

33. Nipkow, T., Paulson, L.C., Wenzel, M.T.: Isabelle/HOL - A Proof Assistant for Higher-Order Logic. LNCS, vol. 2283, p. 218. Springer, Heidelberg (2002)

34. Zuccherato, R., Nystrom, M.: RFC 3163: ISO/IEC 9798-3 Authentication SASL Mechanism (2001), http://www.rfc-editor.org/info/rfc3163 